\title{
Modelo de ecuaciones estructurales con variables influyentes en la calidad de vida urbana. Caso de estudio: ciudad de Cienfuegos, Cuba
}

\author{
Structural equations model with influential variables on the \\ urban quality of life. Case study: Cienfuegos' city, Cuba \\ Daylí Covas Varela ${ }^{1 *} \quad$ Gilberto Dionisio Hernández Pérez ${ }^{2}$ \\ Juan José Cabello Eras ${ }^{3} \quad$ Laura Crespo García ${ }^{4}$ \\ Recibido 04 de septiembre de 2018, aceptado 25 de marzo de 2019 \\ Received: September 04, 2018 Accepted: March 25, 2019
}

\begin{abstract}
RESUMEN
Este artículo presenta un modelo de ecuaciones estructurales que miden la calidad de vida urbana (CVU), tomando como referencia la ciudad de Cienfuegos (Cuba). El objetivo es determinar las variables que influyen en la calidad de vida urbana, para ello se desarrolla un procedimiento que permite el diseño de dos modelos: uno desde la dimensión objetiva y otro desde la dimensión subjetiva de este concepto. Como principal resultado se muestra un modelo de relaciones entre indicadores de gestión, a partir de información suministrada por los decisores locales y otro modelo que relaciona indicadores de percepción, con información dada por los ciudadanos. La comparación entre ambos modelos permite generalizar qué variables, tales como la salud, la vivienda, los ingresos personales y la carga contaminante al medio ambiente son factores determinantes en la CVU de la ciudad.
\end{abstract}

Palabras clave: Calidad de vida urbana, procedimiento, modelo de ecuaciones estructurales.

\begin{abstract}
This paper presents a structural equations model (SEM) that measure the Urban Quality of Life (UQoL), taking as reference Cienfuegos's city $(\mathrm{Cuba})$. The objective is to determine the variables that influence the UQoL, to develop a procedure that allows the design of two models: one from the objective dimension and the other from the subjective dimension of this concept. As main results a model of relations of management indicators, given by local decision makers and another model that relates perception indicators, given by citizens is showed. The comparison between these models makes it possible to generalize that variables such as health, housing and income are determining factors in the UQoL of Cienfuegos's city.
\end{abstract}

Keywords: Urban quality of life, procedure, structural equations model.

1 Universidad de Cienfuegos "Carlos Rafael Rodríguez”. Departamento de Ingeniería Industrial. Cienfuegos, Cuba. E-mail: dcovas@ucf.edu.cu

2 Universidad Central "Marta Abreu" de Las Villas. Departamento de Ingeniería Industrial. Santa Clara, Cuba. E-mail: ghdez@uclv.edu.cu

3 Universidad de la Costa. Departamento de Energía. Barranquilla, Colombia. E-mail: jcabello2@cuc.edu.co

4 Almacenes Universales S.A. Departamento de Recursos Humanos. Cienfuegos, Cuba. E-mail: rhumanos05@cfg.ausa.cu

* Autor de correspondencia: dcovas@ucf.edu.cu 


\section{INTRODUCCIÓN}

El carácter multidisciplinario y multidimensional que se asocia al concepto de Calidad de Vida Urbana (CVU) se remonta a sus orígenes [1] y ha sido destacado por diferentes autores en su evolución y diversidad de aplicaciones en muchas ciudades del mundo [2-5].

El estudio de la CVU se ha concretado en el establecimiento de diferentes y muy diversos indicadores [6] que evalúan dos dimensiones fundamentales: una objetiva (visión cuantitativa y medible) y una subjetiva (enfoque cualitativo basada en la percepción) [4]. Algunos estudios desarrollan individualmente cada una de estas dimensiones o las combinan en la construcción de índices que denotan el estado de este concepto en un contexto urbano determinado $[7,8]$.

El estudio y evaluación de la CVU es frecuentemente utilizado como herramienta de diseño urbano con el objetivo de atender las necesidades de la sociedad y así alcanzar niveles de bienestar para el ciudadano $[9,10]$ o en determinados casos, identificar aquellos espacios "mejores" para vivir $[11,12]$.

Todas las publicaciones antes citadas abordan la CVU y ofrecen modelos compuestos por indicadores que solo son probados desde la práctica. Sin embargo, existen pocas referencias sobre las relaciones que pueden existir entre las variables que evalúan este constructo y su nivel de influencia.

El concepto de modelo de ecuaciones estructurales (SEM por sus siglas en inglés ${ }^{5}$ ) se introdujo en el primer tercio del siglo pasado. Este método desarrolla una forma de desagregar las correlaciones observadas en un sistema de ecuaciones que matemáticamente describan su hipótesis respecto a las relaciones causales, asumiendo la existencia de relaciones lineales y no lineales [13].

Estos modelos permiten proponer el tipo y dirección de las relaciones que se espera encontrar entre las variables contenidas en este. A partir de la estimación de los parámetros que se especifican por las relaciones propuestas a nivel teórico, se

5 SEM: Structural Equation Model confirma el modelo de las relaciones propuestas mediante el análisis de una muestra.

Los estudios sobre la CVU y el desarrollo de modelos para su gestión, dotan a los gobiernos locales de las ciudades, de herramientas de apoyo para la toma de decisiones y la gestión de políticas públicas para el desarrollo de la sociedad, su seguimiento y control.

En Cuba se han publicado muy pocos trabajos orientados al estudio de la CVU $[2,14]$ y aunque ya han transcurrido algo más de 40 años de la aplicación en el país del actual sistema de gobierno, las administraciones municipales no cuentan aún con un método, procedimiento, técnica u herramienta que apoye la toma de decisiones para gestionar la $\mathrm{CV}$ en la ciudad con mayor objetividad [15].

Es por ello que entre los objetivos de la investigación originaria que sustenta la presente contribución, se encuentra el diseñar un modelo de ecuaciones estructurales que permita conocer las relaciones que se establecen entre las variables que inciden en la CVU, como parte de un instrumento metodológico para gestionarla en ciudades de Tipo $\mathrm{I}^{6}$ en Cuba y comprobarlo desde sus dimensiones objetiva y subjetiva, mediante su aplicación en la ciudad de Cienfuegos como caso de estudio práctico representativo de las de su tipo en el país. Esta clasificación de ciudades es independiente del estatus político-administrativo de la ciudad, aunque coincide con casi todas las ciudades capitales provinciales en Cuba. Son las ciudades que presentan una mayor presión demográfica con elevada organización social, política y económica, donde una parte notable de la población vive de actividades no agrícolas y sí de la industria, el comercio y los servicios.

El desarrollo del SEM desde las dimensiones objetiva y subjetiva de la CVU contribuye a determinar los aspectos más importantes de la problemática que se analiza desde una visión global. Además, ayuda a reducir la cantidad de información que debe ser considerada, agrupando las relaciones de un gran número de variables en unos pocos factores, resaltando los aspectos más relevantes del estudio.

6 Ciudades que tienen de 100000 a 499999 habitantes según la Oficina Nacional de Estadística e Información de Cuba. 


\section{ANÁLISIS TEÓRICO}

Según González Rodríguez [16] la CVU depende, en efecto, de las capacidades de los individuos para su realización dentro del medio, pero también de las condiciones presentes en el mismo que posibiliten sus actividades y potencien sus capacidades. El concepto de CVU no posee una lectura homogénea y cada disciplina que lo aborda lo hace desde su propia perspectiva en un contexto específico [17]. Los componentes básicos de la CVU son el individuo, la sociedad, la necesidad y los satisfactores de esa necesidad, todos estos en un contexto urbano y en un momento y tiempo determinado $[4,5]$. Es por ello que se debe resaltar su carácter social, cultural, geográfico, económico, político, ambiental y en general, multidimensional que abarca todas las áreas de la vida.

Algunos estudios la definen como las condiciones presentes en una ciudad que garantizan el confort biológico y psicológico de sus habitantes [16], aunque la mayoría de las investigaciones lo aborda desde las políticas públicas $[18,19]$.

Es frecuente encontrar estudios internacionales de relevancia en esta temática, principalmente en países como Argentina, Chile y Colombia [20-22]; Italia, España y Países Bajos [23-25]; Irán, Indonesia y Malasia [26-28] y Egipto y Nigeria $[29,30]$.

En las dos dimensiones (objetiva y subjetiva) que comprende este concepto, la CVU se evalúa a partir de indicadores sociales (percepción de los ciudadanos, seguridad, arte y cultura, densidad de población), económicos (condiciones de la vivienda, oportunidad de trabajo, costo de la vida, impuestos), ambientales (contaminación, ruido, limpieza), de infraestructura urbana (condiciones de parques, jardines y espacios públicos, transporte, acceso a los servicios) e institucionales (calidad de los servicios de gobierno) [31].

\section{METODOLOGÍA}

Estudios como los realizados por Carbó Carreté [32], Cancino [33] y Hair [34] hacen referencia a diseños de $S E M$ que en general, coinciden en el empleo del procedimiento metodológico propuesto por García Veiga [13] que se muestra en la Figura 1.
Fuente: adaptado de García Veiga [13].

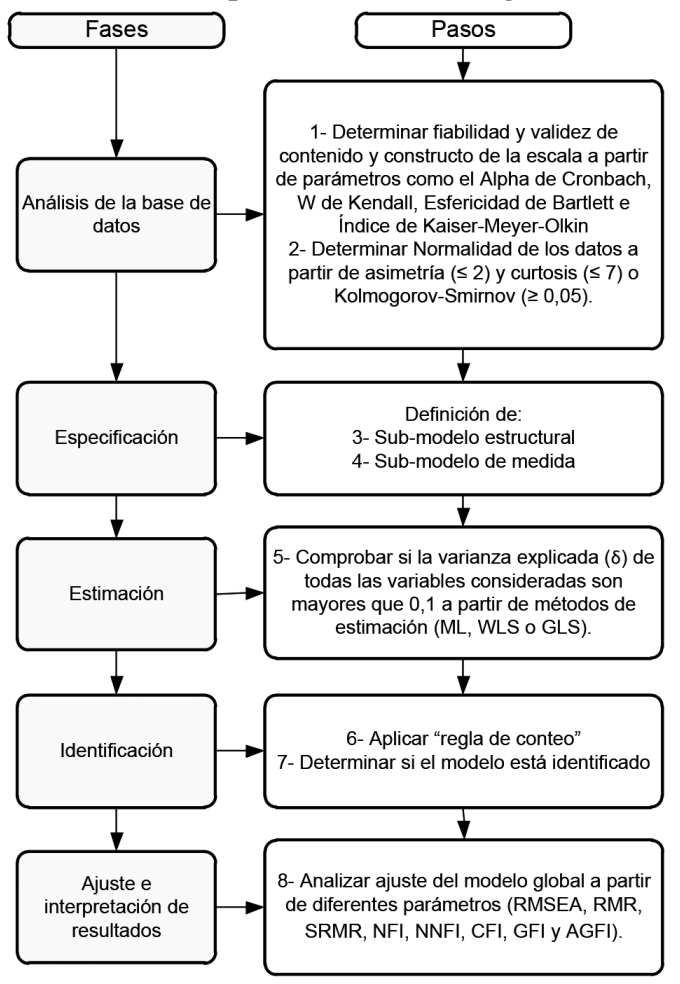

Figura 1. Procedimiento para elaborar un SEM.

La obtención de los SEM en este estudio se realiza a partir del empleo del paquete estadístico Lisrel (Linear Structural Relationship) versión $8.8^{7}$ como uno de los programas más conocidos para el análisis de $S E M$.

\section{Fase: Análisis de la base de datos}

Previo al diseño de un SEM debe analizarse la base de datos que lo soporta para cada aplicación específica. La fuente de la base de datos debe ser descrita y comprobada la normalidad de los datos utilizados, la validez de contenido y de criterio, así como la fiabilidad de los instrumentos que se utilizan para obtenerla. Para ello se diseñan dos cuestionarios: uno para la base de datos de la dimensión objetiva y el otro para el de la subjetiva.

Estos cuestionarios se aplican a una muestra significativa de personas que puedan ser considerados expertos en el tema y que ofrece la información que se necesita en los modelos. Para verificar la

\footnotetext{
7 https://www.updatestar.com
} 
validez de contenido es necesario realizar un estudio de la literatura relevante en el tema y recoger los criterios de los expertos incluidos en la muestra. Esta validez quedará demostrada si el coeficiente "W" de Kendall, calculado a partir de los criterios emitidos por los expertos es $\geq 0,7$, para el que se considera que existe consenso entre estos.

Por su parte, la validez de constructo se determina a través de un análisis factorial de componentes principales [35]. Este análisis se realiza teniendo en cuenta algunos criterios [36] que aceptan la validez de constructo, tales como: la prueba de esfericidad de Bartlett, donde la significación asintótica debe ser menor o igual que 0,05 para demostrar correlación entre las variables; la medida de adecuación de la muestra de Kaiser-Meyer-Olkin (KMO) que considera aceptables valores superiores a 0,5 y que indica la existencia de fuertes relaciones entre las variables.

La fiabilidad del instrumento se determina a partir del coeficiente Alpha de Cronbach, donde se requiere que este sea mayor que 0,7 para considerarse aceptable [35].

Para comprobar la normalidad de los datos utilizados se analiza la curtosis y la asimetría que representan las variables o aplicando la prueba de KolmogorovSmirnov. Para ello y según señalan Curran, West y Finch [37], se considera un comportamiento semejante al normal en índices inferiores a 2 para la asimetría e inferiores a 7 para la curtosis. Para la prueba de Kolmogorov-Smirnov se considera la normalidad de los datos para valores de $\mathrm{P} \geq 0,05$.

Una vez obtenida la base de datos y comprobada su normalidad, validez y fiabilidad, se continúan aplicando las fases de construcción del SEM mostradas en la Figura 1.

\section{Fase: Especificación}

En esta fase se establecen dos sub-modelos: el estructural y el de medida. Estos sub-modelos están compuestos por variables observadas (medidas mediante una encuesta) y latentes (las características o dimensiones estimadas que se pretenden medir con esas variables). A su vez, estas variables pueden ser de dos tipos: endógenas (o dependientes) y exógenas (o independientes).

El sub-modelo estructural (Figura 2) es aquel componente del modelo general que describe

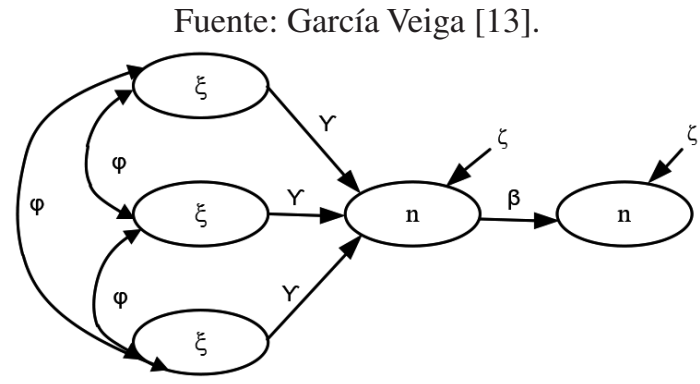

Figura 2. Representación gráfica de un sub-modelo estructural de un SEM.

las relaciones causales entre variables latentes (ya sean endógenas como exógenas) y que se representan gráficamente encerradas en un óvalo; las flechas unidireccionales representan la relación de dependencia entre variables latentes endógenas y las bidireccionales, la existente entre variables latentes exógenas.

La estructura que sigue este tipo de ecuaciones en los sub-modelos estructurales se puede expresar como se muestra en la ecuación (1).

$$
\eta=\beta \eta+{ }_{\gamma} \xi+\zeta
$$

Donde:

$\eta$ : variable endógena latente.

$\xi$ : variable exógena latente.

$\gamma: \quad$ coeficiente que relaciona las variables latentes exógenas $(\xi)$ con las endógenas $(\eta)$.

$\beta$ : coeficiente que relaciona las variables latentes endógenas entre sí.

$\zeta$ : error o término de perturbación; indica que las variables endógenas no son perfectamente determinadas por las ecuaciones estructurales.

Por otra parte, las variables latentes están relacionadas con variables observables a través del sub-modelo de medida (Figura 3), enmarcadas en un rectángulo y que a su vez, se definen por variables endógenas y exógenas a través de las ecuaciones (2) y (3).

$$
\begin{aligned}
& \mathrm{y}=\Lambda \mathrm{y} \eta+\varepsilon \\
& \mathrm{x}=\Lambda \mathrm{x} \xi+\delta
\end{aligned}
$$

Donde:

$\Lambda_{\mathrm{y}}$ : coeficientes de regresión de variables endógenas; representan la carga factorial de estas variables 
Fuente: García Veiga [13].

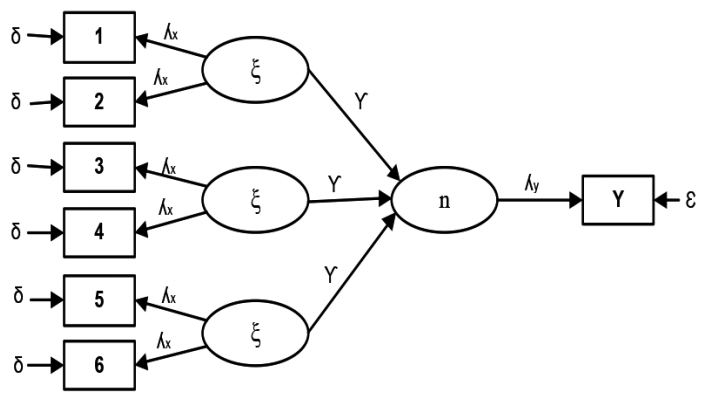

Figura 3. Representación gráfica de un sub-modelo de medida de un SEM.

observables sobre las variables latentes endógenas.

$\Lambda_{\mathrm{x}}$ : coeficientes de regresión de variables exógenas; representan la carga factorial de estas variables observables sobre las variables latentes exógenas.

$\varepsilon$ : errores de predicción de los indicadores endógenos.

$\delta$ : errores de medición de los indicadores exógenos.

\section{Fase: Estimación}

Para la ejecución de esta fase sobre la base de datos específicos de una ciudad, obtenidos a partir de una encuesta $u$ otro instrumento que permita su relevamiento de fuentes primarias, se pueden emplear diferentes métodos de estimación de los parámetros correspondientes a la población muestral considerada, con el objetivo de determinar cuál de estos presenta un mejor ajuste; entre estos pueden señalarse ${ }^{8}$ : máxima verosimilitud $(M L)$, mínimos cuadrados ponderados (WLS) y mínimos cuadrados generalizados $(G L S)$. La aplicación de un software especializado facilita la estimación de estos parámetros por los diferentes métodos que se seleccionen. En esta fase, según plantea García Veiga [13], debe comprobarse que la varianza explicada de las variables $\left(R^{2}\right)$ sea $\geq 0,5$ para una mayor fiabilidad de la medida, aunque el investigador puede fijar su propia medida de fiabilidad.

\section{Fase: Identificación}

Un $S E M$ está identificado si se cumplen las condiciones siguientes: 1 ) el número de parámetros a estimar ( $\mathrm{t}$ ) es menor o igual al número total de

8 Acrónimos en idioma inglés: ML (Maximum Likelihood); WLS (Weighted Least Squares); GLS (Generalized Least Squares). observaciones (S); 2) las variables latentes están asignadas a una escala de medida.

Para verificar el cumplimiento de la primera condición se aplica la conocida como "regla de conteo", donde el número total de variables $(s)$ se determina por la ecuación (4).

$$
s=p+q
$$

Donde:

$p ; q$ : variables endógenas y exógenas consideradas en el estudio, respectivamente.

El número total de observaciones $(S)$ se determina por la ecuación (5).

$$
S=12 s(s+1)
$$

A su vez, el número total de parámetros a ser estimados en el SEM se denota como $t$ y representa la cantidad total de coeficientes, varianzas y covarianzas presentes en el mismo. Entonces, para que un SEM se considere identificado, deberá cumplir con la condicional representada en la ecuación (6).

$$
\mathrm{t} \leq 12 \mathrm{~s}(\mathrm{~s}+1)
$$

Donde:

Si $t=s$, el modelo está identificado.

Si $t<s$, el modelo está sobre identificado.

Si $t>s$, el modelo no está identificado.

\section{Fase: Ajuste e interpretación de resultados}

Una vez que el $S E M$ está identificado y se haya realizado la estimación de sus parámetros, únicamente restan dos pasos que resultan fundamentales para cualquier análisis: su evaluación e interpretación, a fin de contrastar si los datos se han ajustado al modelo propuesto.

Existe un ajuste perfecto del SEM cuando exista una correspondencia perfecta entre la matriz reproducida por el modelo y la matriz de observaciones. Entre los índices identificados como de ajuste global, pueden diferenciarse dos tipos de medida: las medidas absolutas de ajuste y las incrementales que según García Veiga [13] son las que se expresan en los incisos a) y b).

\section{Medidas absolutas de ajuste}

Las medidas absolutas de ajuste determinan el grado en que el modelo global (sub-modelo estructural y 
sub-modelo de medida) predice la matriz inicial de datos. En este grupo de medidas absolutas de ajuste se pueden mencionar'? ${ }^{9}$ la raíz del error de aproximación cuadrático medio (RMSEA), en virtud de la cual la discrepancia entre la matriz reproducida por el modelo y la matriz de observaciones está medida en términos de la población y no en términos de la muestra; el índice de bondad de ajuste $(G F I)$ que aporta información sobre la variabilidad explicada por el modelo; el residuo cuadrático medio $(R M R)$ y su estandarización $(S R M R)$ que muestran la diferencia promedio entre las varianzas y covarianzas predichas y las observadas en el modelo, por lo que un valor pequeño refleja un buen ajuste. Estas medidas resultan de gran utilidad, pues también aportan información sobre la bondad del ajuste absoluto del modelo.

\section{Medidas incrementales de ajuste}

De manera general, los modelos buscan ser aproximaciones simplificadas de la realidad, procurando explicar un comportamiento observado. Con este fin se han desarrollado una serie de indicadores que comparan la mejoría en la bondad de ajuste de un modelo con la correspondiente de un modelo base o nulo (modelo con un ajuste muy pobre).

Dentro esta familia de medidas de ajuste incrementales, las más empleadas son ${ }^{10}$ : el índice de ajuste normado (NFI) que sirve para medir la reducción proporcional en la función de ajuste cuando se pasa del modelo nulo al propuesto; el índice de ajuste no normado (NNFI) que compara el ajuste por grado de libertad del modelo propuesto y nulo; el índice de ajuste comparativo $(C F I)$ que indica un buen ajuste del modelo para valores próximos a la unidad y el índice de bondad de ajuste ajustado (AGFI) que es el mismo GFI, aunque ajustado por los grados de libertad del modelo propuesto y del modelo base o nulo.

En la Tabla 1 se muestran los indicadores de medida de ajuste antes señalados con sus correspondientes

9 Acrónimos en idioma inglés: RMSEA (Root Mean Square Error of Approximation); GFI (Goodness of Fit Index); RMR (Root Mean Residual); SRMR (Standardized Root Mean Residual)

${ }^{10}$ Acrónimos en idioma inglés: NFI (Normed Fix Index); NNFI (Non-Normed Fix Index); CFI (Comparative Fit Index); AGFI (Adjusted Goodness of Fit Index).
Tabla 1. Resumen de los indicadores de medidas de ajustes con sus escalas de aceptación.

\begin{tabular}{|c|c|}
\hline \multicolumn{2}{|c|}{ Valores establecidos } \\
\hline Bueno & Aceptable \\
\hline $0 \leq R M S E A \leq 0,05$ & $0,05 \leq R M S E A \leq 0,08$ \\
\hline $0 \leq R M R \leq 0,06$ & $0,06 \leq R M R \leq 0,10$ \\
\hline $0 \leq S R M R \leq 0,08$ & $0,08 \leq S R M R \leq 0,10$ \\
\hline $0,95 \leq N F I \leq 1,0$ & $0,90 \leq N F I \leq 0,95$ \\
\hline $0,97 \leq N N F I \leq 1,0$ & $0,95 \leq N N F I \leq 0,97$ \\
\hline $0,97 \leq C F I \leq 1,0$ & $0,95 \leq C F I \leq 0,97$ \\
\hline $0,95 \leq G F I \leq 1,0$ & $0,90 \leq G F I \leq 0,95$ \\
\hline $0,90 \leq A G F I \leq 1,0$ & $0,85 \leq A G F I \leq 0,90$ \\
\hline
\end{tabular}

Fuente: García Veiga [13].

rangos de aceptación de la escala en dos niveles: de buen ajuste y de ajuste aceptable para su empleo en casos específicos.

\section{RESULTADOS}

El procedimiento específico para obtener el SEM antes expuesto (Figura 1) y descrito, se incorporó como parte de un procedimiento metodológico general alternativo para la gestión de la CVU por los gobiernos locales en ciudades del tipo I en Cuba y forma parte de la investigación originaria que sustenta la presente contribución, tomando a la ciudad de Cienfuegos como caso de estudio práctico para refrendar la validez de las variables que determinan la dimensión objetiva y subjetiva de la CVU. Su aplicación a este caso se expone a continuación.

\section{Fase: Análisis de la base de datos}

Se analizó y determinó que para conformar el SEM correspondiente a este caso se construyera una base de datos para cada dimensión. Así, para el caso de la dimensión objetiva de la CVU se aplicó un cuestionario a una muestra de 60 delegados (decisores del gobierno) a la Asamblea Municipal del Poder Popular (AMPP) de Cienfuegos como caso de estudio representativo de las 12 ciudades de tipo I en Cuba, sobre la base de las variables que caracterizan esta dimensión y que forman parte de un índice sintético que la caracteriza.

Para la dimensión subjetiva de la CVU se diseñó y aplicó también un cuestionario específico con el objetivo de determinar las variables que evalúan la 
CVU percibida por los ciudadanos de una muestra estratificada de carácter aleatorio de 766 personas residentes en los 13 consejos populares urbanos del municipio de Cienfuegos.

Para probar la validez de contenido, constructo y fiabilidad de los instrumentos utilizados se sometieron ambos a las pruebas ya descritas ("W" de Kendall, KMO, esfericidad de Bartlett y Alpha de Cronbach). De ello resultó que para la dimensión objetiva y subjetiva, la prueba no paramétrica "W" de Kendall dio como resultado 0,851 y 0,739 , respectivamente, por lo que el juicio de los expertos se consideró consistente en ambos casos.

En el análisis factorial para la validez de constructo, resalta un valor de KMO de 0,797 y 0,715 (en ambos casos $>0,5)$ y una significación asintótica de la prueba de esfericidad de Bartlett de 0,000 (en ambos casos resultó $<0,05$ ).

El Alpha de Cronbach arrojó valores de 0,748 y 0,724 (en ambos casos $>0,7$ ), por lo que se pudo concluir que el análisis factorial era conveniente y aceptable, y por tanto, los instrumentos que medían la dimensión objetiva y subjetiva de la CVU eran válidos y fiables. Los resultados de sus aplicaciones se muestran en la Tabla 2 para las variables que caracterizan la dimensión objetiva y en la Tabla 3 para las que caracterizan la dimensión subjetiva.

Posteriormente se procedió al análisis descriptivo de los datos obtenidos de la aplicación de los cuestionarios en la población muestral seleccionada, para luego someter a los modelos propuestos (objetivo y subjetivo) a las pruebas de curtosis y asimetría para probar su normalidad univariada. Para ello se utilizó el programa estadístico Statgraphics Centurion XVI. II Profesional $X V^{11}$, cuyos resultados se muestran en las Tablas 4 y 5.

De acuerdo con los valores estadísticos obtenidos, se pudo comprobar que existe normalidad univariada de los datos, toda vez que cumplen con las condiciones de asimetría y curtosis en el rango establecido $[-2 ; 2]$.

\section{Fase: Especificación}

En el modelo de la dimensión objetiva se utilizó la matriz de datos originales, obtenida a partir de

${ }^{11} \mathrm{https} / /$ www.portalprogramas.com/statgraphics/
Tabla 2. Variables y su codificación que caracterizan la dimensión objetiva de la CVU en ciudades del tipo I en Cuba.

\begin{tabular}{|c|c|}
\hline \multicolumn{2}{|c|}{ 1. Dimensión social } \\
\hline Indicadores & Nomenclatura \\
\hline Educación & Soc1 \\
\hline Salud & Soc2 \\
\hline Cultura & Soc3 \\
\hline Deporte & Soc4 \\
\hline Seguridad ciudadana & Soc5 \\
\hline Demografía & Soc6 \\
\hline \multicolumn{2}{|c|}{ Dimensión de infraestructura urbana } \\
\hline Viales & Urb1 \\
\hline Energía eléctrica & Urb2 \\
\hline Acueducto y alcantarillado & Urb3 \\
\hline Comunicaciones & Urb4 \\
\hline Transporte & Urb5 \\
\hline Vivienda & Urb6 \\
\hline \multicolumn{2}{|c|}{ Dimensión económica } \\
\hline Ingresos personales & Eco1 \\
\hline Actividad económica & Eco2 \\
\hline Ingresos públicos & Eco3 \\
\hline Empleo & Eco4 \\
\hline Actividad tributaria & Eco5 \\
\hline Innovaciones & Eco6 \\
\hline Inversiones & Eco7 \\
\hline \multicolumn{2}{|c|}{ Dimensión ambiental } \\
\hline Carga contaminante dispuesta & Amb1 \\
\hline Áreas verdes & Amb2 \\
\hline Inversiones para la protección & Amb3 \\
\hline Recolección de desechos sólidos & Amb4 \\
\hline Reciclaje & Amb5 \\
\hline Limpieza urbana & Amb6 \\
\hline Energía por recursos renovables & Amb7 \\
\hline \multicolumn{2}{|c|}{ Percepción general } \\
\hline CVU objetiva & Y1 \\
\hline
\end{tabular}

haber relevado la información necesaria mediante la aplicación del correspondiente cuestionario (con todas las variables observadas) a 60 decisores del municipio de Cienfuegos. En la Figura 4 se muestra el modelo general obtenido de la dimensión objetiva.

En el modelo general de la dimensión objetiva son:

\section{Variables observadas:}

Exógenas: Soc1, Soc2, Soc3, Soc4, Soc5, Soc6, Urb1, Urb2, Urb3, Urb4, Urb5, Urb6, Eco1, Eco2, Eco3, Eco4, Eco5, Eco6, Eco7, Amb1, Amb2, Amb3, Amb4, Amb5, Amb6 y Amb7 
Tabla 3. Variables de la dimensión subjetiva identificadas por la población encuestada.

\begin{tabular}{|c|c|}
\hline \multicolumn{2}{|c|}{ 1. Dimensión social } \\
\hline Indicadores & Nomenclatura \\
\hline Educación & $\mathrm{X} 1$ \\
\hline Salud & $\mathrm{X} 2$ \\
\hline Cultura & $\mathrm{X} 3$ \\
\hline Deporte & $\mathrm{X} 4$ \\
\hline Seguridad ciudadana & $\mathrm{X} 5$ \\
\hline \multicolumn{2}{|c|}{ Dimensión de infraestructura urbana } \\
\hline Telefonía pública & X6 \\
\hline Transporte privado & $\mathrm{X} 7$ \\
\hline Acueducto y alcantarillado & $\mathrm{X} 8$ \\
\hline Telefonía privada & $\mathrm{X} 9$ \\
\hline Transporte público & $\mathrm{X} 10$ \\
\hline Vivienda & X11 \\
\hline Conectividad & $\mathrm{X} 12$ \\
\hline Viales & $\mathrm{X} 13$ \\
\hline Energía eléctrica & $\mathrm{X} 14$ \\
\hline \multicolumn{2}{|c|}{ Dimensión económica } \\
\hline Ingresos personales & $\mathrm{X} 15$ \\
\hline \multicolumn{2}{|l|}{ Dimensión ambiental } \\
\hline Carga contaminante & $\mathrm{X} 16$ \\
\hline \multicolumn{2}{|c|}{ Percepción general ciudadana } \\
\hline CVU subjetiva & Y1 \\
\hline
\end{tabular}

\section{Endógena: Y1}

\section{Variables latentes:}

Exógenas: DS $\left(\xi_{\mathrm{S}}\right), \mathrm{DIU}\left(\xi_{\mathrm{U}}\right), \mathrm{DE}\left(\xi_{\mathrm{E}}\right)$ y DA $\left(\xi_{\mathrm{M}}\right)$ Endógenas: ICVUo $\left(\eta_{\text {ICVUo }}\right)$

\section{Errores de medida:}

De variables observadas exógenas: $\delta_{1}, \delta_{2}, \delta_{3}, \delta_{4}$, $\delta_{5}, \delta_{6}, \delta_{7}, \delta_{8}, \delta_{9}, \delta_{10}, \delta_{11}, \delta_{12}, \delta_{13}, \delta_{14}, \delta_{15}, \delta_{16}, \delta_{17}$, $\delta_{18}, \delta_{19}, \delta_{20}, \delta_{21}, \delta_{22}, \delta_{23}, \delta_{24}, \delta_{25} \psi \delta_{26}$

De variables observadas endógenas: $\varepsilon_{1}$

Términos de perturbación: $\zeta_{1}$; incluye los efectos de las variables omitidas, los errores de medida y la aleatoriedad del proceso especificado.

Coeficientes de regresión: $\lambda_{\mathrm{x}}, \lambda_{\mathrm{y}}$; relacionan las variables latentes con las observadas.

Coeficientes de regresión: $\gamma_{11}, \gamma_{12}, \gamma_{13} \psi \gamma_{14}$; representan la relación entre una variable latente exógena y una endógena. Además de $\phi_{12}, \phi_{13}, \phi_{14}$, $\phi_{23}, \phi_{24}$ y $\phi_{34}$, como la covariación entre las variables latentes exógenas.
Tabla 4. Estadísticos descriptivos de la dimensión objetiva.

\begin{tabular}{|l|c|c|}
\hline \multicolumn{1}{|c|}{ Variable } & Asimetría & Curtosis \\
\hline Educación & $-0,740$ & $-0,752$ \\
\hline Salud & $-0,831$ & $-0,576$ \\
\hline Cultura & 0,209 & $-1,037$ \\
\hline Deporte & $-1,015$ & 0,58 \\
\hline Seguridad ciudadana & 0.509 & -0.970 \\
\hline Demografía & 0,534 & $-1,071$ \\
\hline Viales & $-0,426$ & $-0,990$ \\
\hline Energía eléctrica & $-1,396$ & 0,220 \\
\hline Acueducto y alcantarillado & $-0,438$ & $-0,738$ \\
\hline Comunicaciones & $-0,129$ & $-1,582$ \\
\hline Transporte & $-1,835$ & 1,413 \\
\hline Vivienda & $-0,847$ & $-0,063$ \\
\hline Ingresos personales & $-0,014$ & $-1,188$ \\
\hline Actividad económica & $-0,037$ & $-0,859$ \\
\hline Ingresos públicos & 0,256 & $-0,831$ \\
\hline Empleo & 0,032 & $-0,568$ \\
\hline Actividad tributaria & $-0,031$ & $-0,265$ \\
\hline Innovaciones & 0,419 & $-1,889$ \\
\hline Inversiones & 0,419 & $-1,889$ \\
\hline Carga contaminante dispuesta & 0,137 & $-1,051$ \\
\hline Áreas verdes & 0,206 & $-1,026$ \\
\hline Inversiones para la protección & 0,488 & $-0,721$ \\
\hline Recolección de desechos sólidos & 0,510 & $-0,990$ \\
\hline Reciclaje & 0,473 & $-0,674$ \\
\hline Limpieza urbana & $-0,740$ & $-0,752$ \\
\hline Energía por recursos renovables & $-0,831$ & $-0,576$ \\
\hline CVU objetiva & $-1,015$ & 0,58 \\
\hline & &
\end{tabular}

Tabla 5. Estadísticos descriptivos de la dimensión subjetiva.

\begin{tabular}{|l|c|c|}
\hline \multicolumn{1}{|c|}{ Variables } & Asimetría & Curtosis \\
\hline Educación & $-0,279$ & $-1,275$ \\
\hline Salud & $-0,220$ & $-1,215$ \\
\hline Cultura & $-0,520$ & $-1,157$ \\
\hline Deporte & $-0,700$ & $-0,472$ \\
\hline Seguridad ciudadana & -0.224 & -0.909 \\
\hline Telefonía pública & $-0,436$ & $-1,081$ \\
\hline Transporte privado & $-0,585$ & $-0,712$ \\
\hline Acueducto y alcantarillado & $-0,944$ & $-0,228$ \\
\hline Telefonía privada & $-0,645$ & $-0,767$ \\
\hline Transporte público & $-0,616$ & $-0,695$ \\
\hline Vivienda & $-0,361$ & $-1,027$ \\
\hline Conectividad & $-0,203$ & 0,894 \\
\hline Viales & $-0,718$ & $-0,495$ \\
\hline Energía eléctrica & $-1,009$ & 1,761 \\
\hline Ingresos personales & $-0,459$ & $-1,119$ \\
\hline Carga contaminante & $-0,130$ & $-0,838$ \\
\hline CVU subjetiva & $-0,332$ & $-0,894$ \\
\hline
\end{tabular}




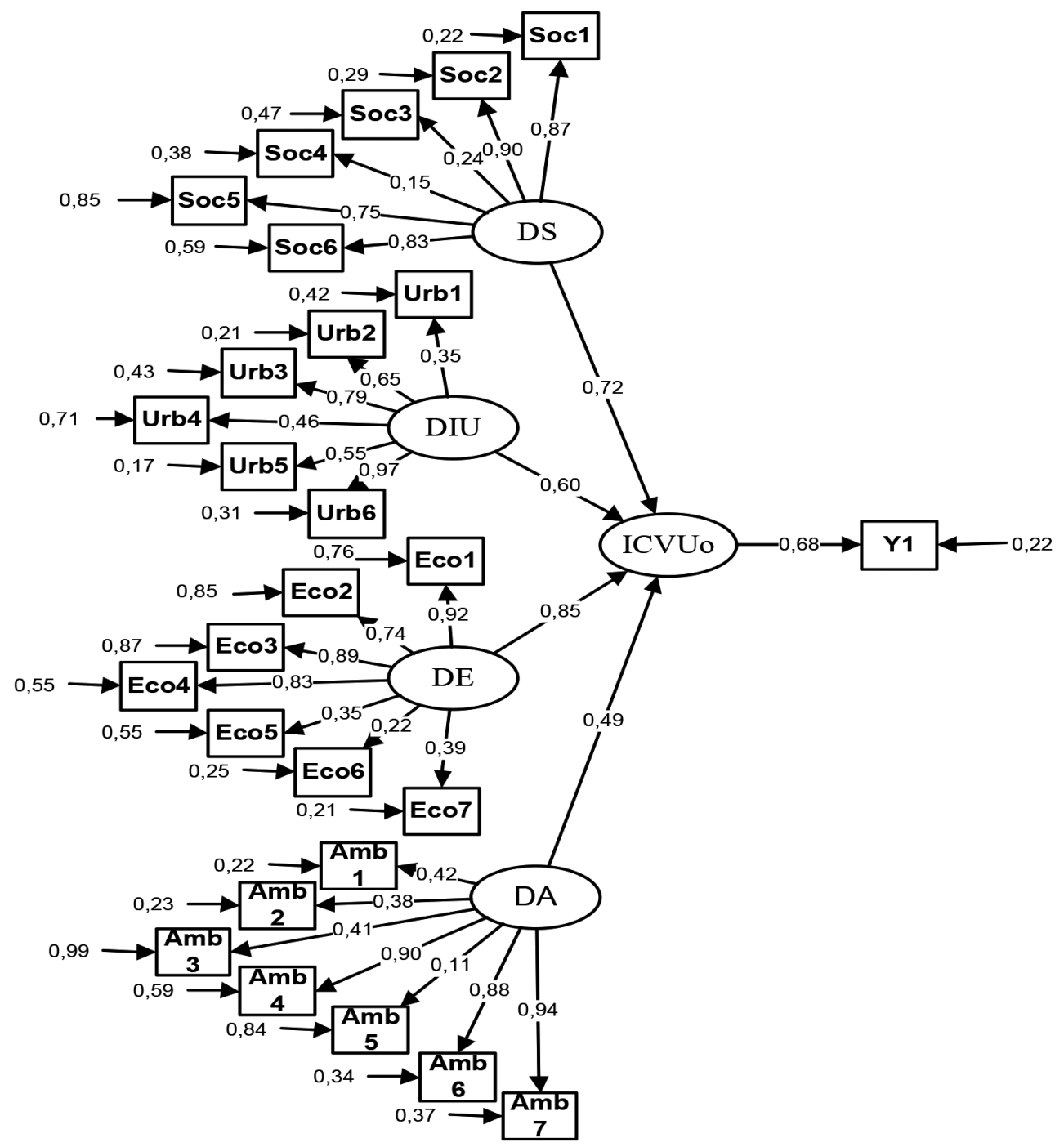

Figura 4. Modelo de medida global estimado de la dimensión objetiva.

Para la dimensión subjetiva que se muestra en la Figura 5, se construye el SEM en el que se definen 22 variables (17 observadas y 5 latentes).

En el modelo general de la dimensión subjetiva son:

\section{Variables observadas:}

Exógenas: X1, X2, X3, X4, X5, X6, X7, X8, X9, X10, X11, X12, X13, X14, X15 y X16

Endógena: $\mathrm{Y} 1$

\section{Variables latentes:}

Exógenas: DS $\left(\xi_{\mathrm{S}}\right)$, DIU $\left(\xi_{\mathrm{U}}\right), \mathrm{DE}\left(\xi_{\mathrm{E}}\right)$ y DA $\left(\xi_{\mathrm{M}}\right)$. Endógenas: ICVUs $\left(\eta_{\mathrm{ICVUS}}\right)$

\section{Errores de medida:}

De variables observadas exógenas: $\delta_{1}, \delta_{2}, \delta_{3}, \delta_{4}, \delta_{5}$, $\delta_{6}, \delta_{7}, \delta_{8}, \delta_{9}, \delta_{10}, \delta_{11}, \delta_{12}, \delta_{13}, \delta_{14}, \delta_{15}$ y $\delta_{16}$ De variables observadas endógenas: $\varepsilon_{1}$

Términos de perturbación: $\zeta_{1}$; incluye los efectos de las variables omitidas, los errores de medida y la aleatoriedad del proceso especificado.

Coeficientes de regresión: $\lambda_{\mathrm{x}}, \lambda_{\mathrm{y}}$; relacionan las variables latentes con las observadas.

Coeficientes de regresión: $\gamma_{11}, \gamma_{12}, \gamma_{13}$ y $\gamma_{14}$; representan la relación entre una variable latente 


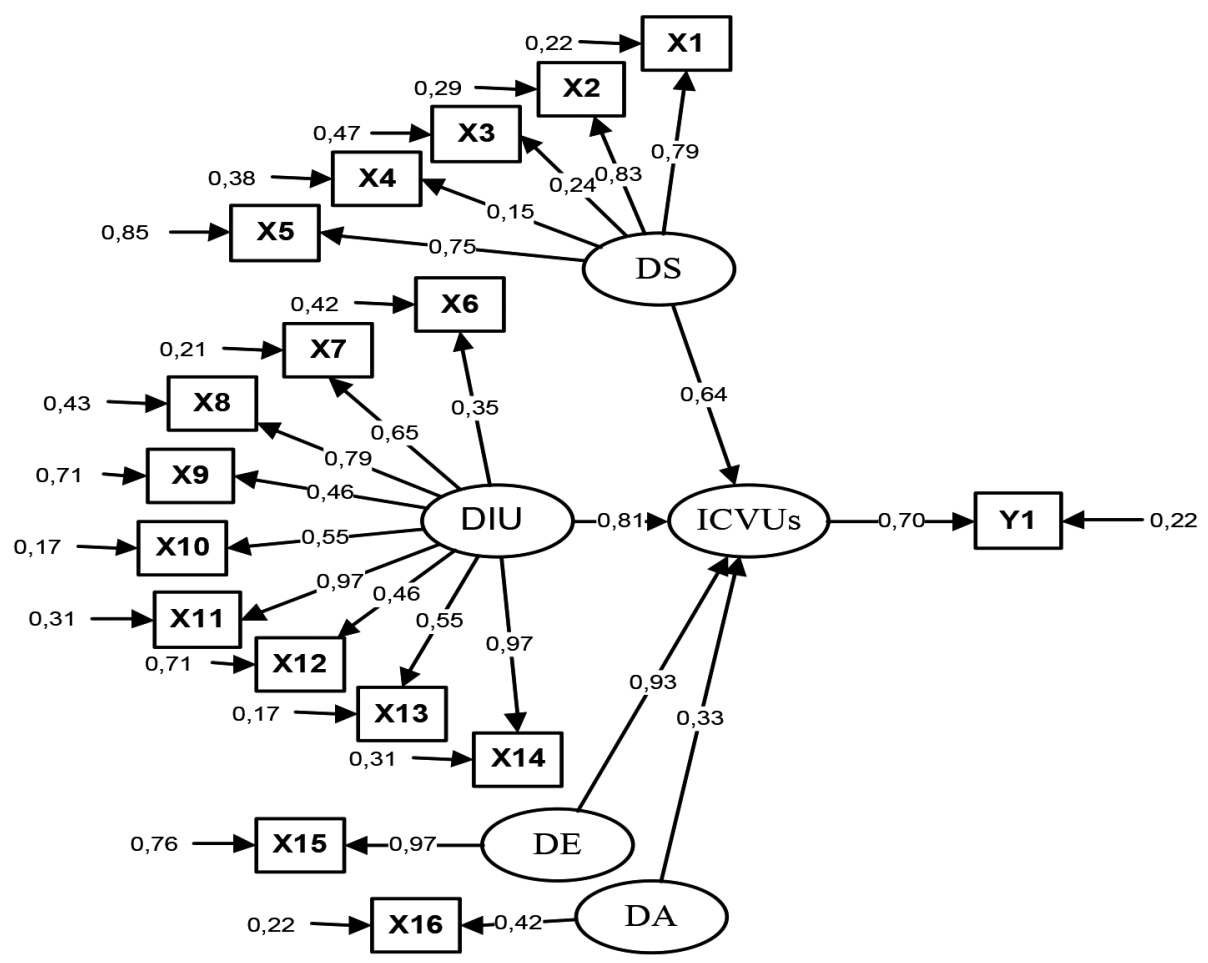

Figura 5. Modelo de medida global estimado de la dimensión subjetiva.

exógena y una endógena. Además de $\phi_{12}, \phi_{13}, \phi_{14}$, $\phi_{23}, \phi_{24}$ y $\phi_{34}$, como la covariación entre las variables latentes exógenas.

\section{Fase: Estimación}

En esta fase se analizaron las relaciones entre las diferentes variables del modelo inicial de medida, tanto de la dimensión objetiva como de la subjetiva mediante el método de máxima verosimilitud $(M L)$. Se fija un valor de $\mathrm{R}^{2}>0,1$ para aceptar la varianza explicada de cada una de las variables, pudiéndose comprobar (Tabla 6) que los $\mathrm{R}^{2}$ de todas las variables consideradas son mayores que 0,1 en todos los casos, cumpliendo así con la condición señalada.

$\mathrm{Al}$ analizar los resultados de las variables latentes de la dimensión objetiva en el modelo estructural (Figura 6) se observa que existe una fuerte correlación entre las dimensiones social-urbana, social-económica y urbana-económica; moderada entre las dimensiones social-ambiental y económica-ambiental, mientras que entre las dimensiones urbana-ambiental, aunque fuerte, la correlación es inversa.
De igual manera se procedió en el análisis de los resultados de las variables latentes de la dimensión subjetiva en el sub-modelo estructural que se presenta en la Figura 7, donde se aprecia también que existe una fuerte correlación entre las dimensiones social y urbana, moderada entre la social y la económica y entre la urbana y la económica, así como baja entre la social y la ambiental y entre las urbana y medioambiental y económica y ambiental, aunque inversa en estos dos últimos casos.

\section{Fase: Identificación}

Es en esta fase del procedimiento que se identifica el SEM para ambas dimensiones (objetiva y subjetiva). Así y utilizando la "regla de conteo" de la dimensión objetiva, se determinó que:

Total de variables endógenas observadas; $p=1$. Total de variables exógenas observadas; $q=26$.

Como que en el total de las 27 variables observadas $(s)$, el número de parámetros a ser estimados $(t)$ de 64 es menor que el número de 
Tabla 6. Varianza explicada de cada una de las variables $\left(\mathrm{R}^{2}\right)$.

\begin{tabular}{|c|c|c|c|c|}
\hline & \multicolumn{4}{|c|}{ Valores de $\mathbf{R}^{2}$} \\
\hline & Social & Urbana & Económica & Ambiental \\
\hline Dimensión objetiva & $\begin{array}{l}\text { Soc1 }=0,844 \\
\text { Soc2 }=0,942 \\
\text { Soc3 }=0,290 \\
\text { Soc4 }=0,210 \\
\text { Soc5 } 5=0,658 \\
\text { Soc6 }=0,782\end{array}$ & $\begin{aligned} \text { Urb1 } & =0,593 \\
\text { Urb2 } & =0,625 \\
\text { Urb3 } & =0,752 \\
\text { Urb4 } & =0,312 \\
\text { Urb5 } & =0,236 \\
\text { Urb6 } & =0,967\end{aligned}$ & $\begin{array}{l}\text { Eco1 }=0,977 \\
\text { Eco2 }=0,733 \\
\text { Eco3 }=0,832 \\
E \operatorname{co} 4=0,899 \\
E \operatorname{co} 5=0,541 \\
E \operatorname{co6}=0,305 \\
E \operatorname{co} 7=0,432\end{array}$ & $\begin{array}{l}\text { Amb1 }=0,552 \\
\text { Amb2 }=0,321 \\
\text { Amb3 }=0,571 \\
\text { Amb4 }=0,751 \\
\text { Amb5 }=0,295 \\
\text { Amb6 }=0,681 \\
\text { Amb7 }=0,821\end{array}$ \\
\hline Dimensión subjetiva & $\begin{array}{l}X 1=0,775 \\
X 2=0,870 \\
X 3=0,457 \\
X 4=0,212 \\
X 5=0,705\end{array}$ & $\begin{aligned} \mathrm{X} 6 & =0,263 \\
\mathrm{X} 7 & =0,616 \\
\mathrm{X} 8 & =0,662 \\
\mathrm{X} 9 & =0,490 \\
\mathrm{X} 10 & =0,580 \\
\mathrm{X} 11 & =0,897 \\
\mathrm{X} 12 & =0,445 \\
\mathrm{X} 13 & =0,527 \\
\mathrm{X} 14 & =0,970\end{aligned}$ & $\mathrm{X} 15=0,868$ & $\mathrm{X} 16=0,819$ \\
\hline
\end{tabular}

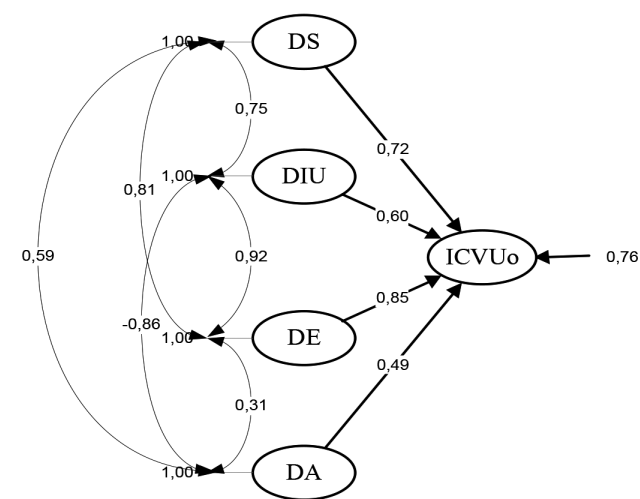

Figura 6. Modelo estructural de la dimensión objetiva.

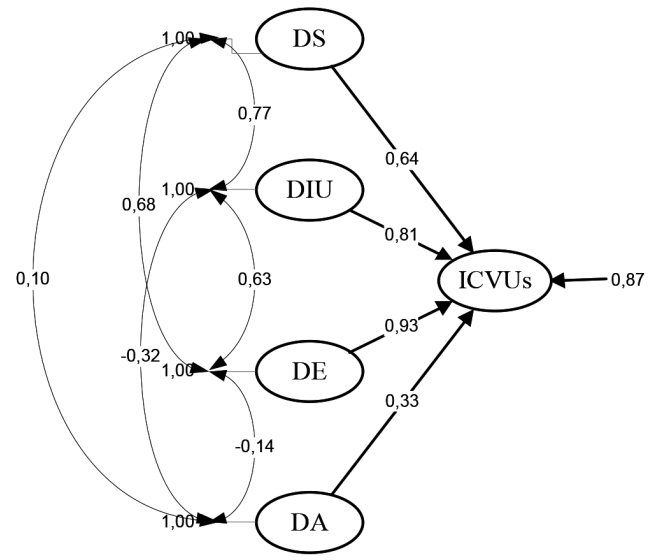

Figura 7. Modelo estructural de la dimensión subjetiva. observaciones totales (S) de 378, el SEM cumple con la condición necesaria para estar identificado (sobre-identificado en este caso). Similar análisis se realizó para el SEM de la dimensión subjetiva, determinándose que:

Total de variables endógenas observadas; $p=1$. Total de variables exógenas observadas; $q=16$.

Por tanto y en este caso, como del total de 17 variables observadas $(s)$, el número de parámetros a ser estimados $(t)$ de 67 es también menor que el número de observaciones totales $(S)$ de 153 , el modelo cumple con la condición necesaria para estar identificado (también sobre-identificado).

Fase: Ajuste e interpretación de los resultados Correspondió en esta fase evaluar e interpretar los diferentes indicadores que miden el ajuste de los modelos de la dimensión objetiva y subjetiva que se muestran en la Tabla 7. Al comparar los valores de estos indicadores con los valores establecidos de aceptación que se exponen en la Tabla 1, se puede considerar que el ajuste es en general aceptable, al encontrarse todos los indicadores dentro de los límites de aceptación establecidos.

Los modelos estructural y de medida de la dimensión objetiva quedaron expresados mediante las ecuaciones (7) y (8), respectivamente: 
Tabla 7. Resumen de los indicadores de medidas de ajuste de los SEM de las dimensiones objetiva y subjetiva para la ciudad de Cienfuegos.

\begin{tabular}{|c|c|c|}
\hline \multirow{2}{*}{ Indicadores de medidas de ajustes } & \multicolumn{2}{|c|}{ Dimensiones } \\
\cline { 2 - 3 } & Objetiva & Subjetiva \\
\hline RMSEA & 0,077 (aceptable) & 0,080 (aceptable) \\
\hline RMR & 0,100 (aceptable) & 0,038 (bueno) \\
\hline SRMR & 0,090 (aceptable) & 0,076 (bueno) \\
\hline NFI & 0,920 (aceptable) & 0,900 (aceptable) \\
\hline NNFI & 0,965 (aceptable) & 0,970 (aceptable) \\
\hline CFI & 0,970 (bueno) & 0,972 (bueno) \\
\hline GFI & 0,910 (aceptable) & 0,920 (aceptable) \\
\hline AGFI & 0,850 (aceptable) & 0,880 (aceptable) \\
\hline
\end{tabular}

Modelo estructural:

$$
\begin{gathered}
\text { IICVUo }=0,72 * \xi D S+0,60 * \\
\xi D I U+0,85 * \xi D E+0,49 * \xi D A+0,76
\end{gathered}
$$

\section{Modelo de medida indicador endógeno:}

$$
\mathrm{Y} 1=0,68 * \eta \mathrm{ICVUo}+0,227
$$

A su vez, los modelos estructural y de medida de la dimensión subjetiva quedaron expresados mediante las ecuaciones (9) y (10), respectivamente. Modelo estructural:

$$
\begin{gathered}
\text { ПICVUo }=0,64 * \xi D S+0,81 * \xi D I U+ \\
0,93 * \xi D E+0,33 * \xi D A+0,87
\end{gathered}
$$

Modelo de medida indicador endógeno:

$$
\mathrm{Y} 1=0,70 * \eta \mathrm{ICVUs}+0,22
$$

Luego de analizar los SEM de las dimensiones objetiva y subjetiva de la CVU, se resume a continuación una comparación realizada entre los resultados obtenidos asociados a ambas dimensiones para la ciudad objeto de estudio.

Las variables de ambas dimensiones cumplen con el supuesto de la normalidad univariada para la asimetría y la curtosis, así como con la condición de que la varianza explicada de todas las variables sea mayor que 0,1 . Además, ambos modelos presentan un ajuste aceptable. La variable dentro de la dimensión social que más información aporta a su variable latente, tanto para la visión objetiva como para la subjetiva, es la salud. Esto significa que, tanto para los decisores como para los ciudadanos este servicio constituye un factor determinante en la CVU. Por su parte, en la dimensión urbana la variable que más información aporta es la vivienda, por lo que constituye un factor determinante en la CVU al coincidir con la variable de mayor significación en las dos visiones: objetiva y subjetiva. En cuanto a la dimensión económica, tanto los decisores como los ciudadanos reconocen los ingresos personales como un factor determinante en la CVU. La variable energía generada por recursos renovables resulta también decisiva para los decisores en la dimensión ambiental, aunque el ciudadano solo reconoce una variable: carga contaminante, sobre la que centra su percepción de la CVU en esta dimensión.

Cabe señalar que en ambos casos, las correlaciones directas más destacables se establecen entre las dimensiones social y urbana, social y económica, y urbana y económica, además de la relación inversa que también en ambos casos se establece entre las dimensiones urbana y ambiental. Esta relación inversa refleja que el aumento de indicadores urbanos de transporte, energía eléctrica y viales, por solo citar algunos ejemplos, tienen un impacto directo en la disminución de las condiciones del medio ambiente.

Mediante esta comparación entre la visión objetiva y subjetiva de la CVU queda comprobada la alineación que debe existir entre la gestión del gobierno y su impacto en la ciudadanía, al definir las prioridades del Gobierno en lo que resulta importante para el ciudadano. Así, los recursos disponibles por los decisores locales para mejorar la $\mathrm{CV}$ deberán dirigirse hacia la satisfacción ciudadana, contribuyendo con ello a cerrar la "brecha" entre la administración 
pública en función de la $\mathrm{CV}$ y su percepción por la población en función de sus necesidades.

\section{CONCLUSIONES}

Las relaciones causales que se establecen entre las variables que determinan la visión objetiva y subjetiva de la calidad de vida urbana permiten validar un modelo de evaluación de este constructo a través de indicadores concretos mediante el uso de modelos de ecuaciones estructurales.

Los modelos de las visiones objetiva y subjetiva permiten retroalimentar las políticas públicas de los gobiernos locales, al dotar a estos de herramientas de información más robustas y pertinentes para la toma de decisiones en función de lograr la satisfacción ciudadana con un uso eficiente de los recursos disponibles.

Existen indicadores, como por ejemplo la salud, la vivienda y los ingresos personales en la ciudad objeto de estudio que como resultado de la investigación, se revelan como factores determinantes en la calidad de vida urbana, tanto para los decisores como para los ciudadanos, lo que permite dirigir la gestión hacia ambos intereses y prioridades.

\section{AGRADECIMIENTOS}

Los autores desean agradecer al grupo de decisores y ciudadanos que formaron parte de esta investigación, así como a los colaboradores de la Universidad de Cienfuegos "Carlos Rafael Rodríguez" y la Universidad Central "Marta Abreu" de Las Villas por su cooperación.

\section{REFERENCIAS}

[1] N.B. Wish. "Are We Really Measuring the Quality of Life? Well-being Has Subjective Dimensions, As Well As Objective Ones". American Journal of Economics and Sociology. Vol. 45, Issue 1, pp. 93-99. 1986. ISSN: 1536-7150. URL: https://onlinelibrary. wiley.com/doi/abs/10.1111/j.1536-7150.1986. tb01906.x. Fecha de consulta: 10-05-2018. DOI: 10.1111/j.1536-7150.1986.tb01906.x.

[2] J.J. Cabello Eras, D. Covas Varela, G.D. Hernández Pérez, A. Sagastume Gutiérrez, D. García Lorenzo, C. Vandecasteele and L. Hens. "Comparative Study of the Urban
Quality of Life in Cuban First-level Cities from an Objective Dimension". Environment, Development and Sustainability. Vol. 16, Issue 1, pp. 195-215. 2013. ISSN: 1387-585X. URL: http://link.springer.com/article/10.1007/ s10668-013-9470-0. Fecha de consulta: 1406-2016. DOI: 10.1007/s10668-013-9470-0

[3] C. Discoli, C. Ferreyro, G.A. San Juan, L. Dicroce, I. Martini, D.A. Barbero y J. Esparza. "Metodología para la evaluación de la calidad de vida urbana". Bitácora Urbano Territorial. Vol. 17, Issue 2, pp. 95-112. 2010. ISSN: 0124-7913. DOI: 10.1016/j.scs.2013.08.002

[4] J. Gómez Piñeiro. "Aproximación al sistema de indicadores de calidad de la vida urbana". Investigación y espacio, $\mathrm{N}^{\mathrm{o}} 32$, pp. 281-299. 2009. ISSN: 0211-5891. URL: http://www.ingeba.org/lurralde/lurranet/ lur32/32pineiro/32pineiro.pdf.pdf. Fecha de consulta: 10-05-2018

[5] G. Leva. "Indicadores de calidad de vida urbana. Teoría y metodología". Habitat Metropolis. 1ra Edición. Vol. 1, pp. 1-101. Quilmes, Argentina. ISBN: 45382-4288. 2005.

[6] V.M. Rodríguez Espinosa y M. Gómez Delgado. "Propuesta metodológica para la elaboración de un índice de calidad de vida urbana mediante SIG”. Serie Geográfica - Profesora María de los Ángeles Díaz Muñoz, In Memoriam, No 14, pp. 207-221. 2008. ISSN: 1136-5277. URL: http://hdl.handle.net/10017/2085 Fecha de consulta: 10-05-2018

[7] A. Orellana. "Informe final. Calidad de Vida Urbana”. Universidad Católica de Chile. Santiago, Chile. 2011.

[8] S. Lotfi and K. Solaimani. "An assessment of Urban Quality of Life by Using Analytic Hierarchy Process Approach. (Case Study: Comparative Study of Quality of Life in the North of Iran)". Journal of Social Sciences. Vol. 5, Issue 2, pp. 123-133. 2009. ISSN: 1549-3652. URL: http://thescipub.com/ pdf/10.3844/jssp.2009.123.133. Fecha de consulta: 10-05-2018. DOI: 10.3844/ jssp.2009.123.133

[9] M. Momeni, H. Azizi and M. Taghinia. “Urban Management Based on Citizen Participation to Enhance Quality of Life in Tehran". REAL CORP 2011 CHANGE FOR STABILITY: Lifecycles of Cities and Regions, pp. 10791085. 2011. ISBN: 978-3-9503110-1-3. 
[10] B.J. Cartay Angulo. "Consideraciones en torno a los conceptos de calidad de vida y calidad ambiental”. Revista Venezolana de Sociología y Antropología. FERMENTUM. Vol. 14 No 41, pp. 491-502. 2004. ISSN: 0798-3069. URL: www.redalyc.org/pdf/705/70504104. pdf. Fecha de consulta: 10-05-2018

[11] M. Hogan, K. Leyden, R. Conway, A. Goldberg, D. Walsh and P. McKenna-Plumley. "Happiness and Health Across the Lifespan in Five Major Cities: The Impact of Place and Government Performance". Social Science \& Medicine. Vol. 162, Issue August 2016, pp. 168-176. 2016. ISSN: 0277-9536. URL: http://www.sciencedirect.com/science/ article/pii/S0277953616303239. Fecha de consulta: 23-05-2017. DOI: 10.1016/j. socscimed.2016.06.030

[12] P.Y. Sewo, E. Ito and R.A. Carvalho. " The Association of Activity and Participation with Quality of Life between Japanese Older Adults Living in Rural and Urban Areas". Journal of Clinical Gerontology and Geriatrics. Vol. 4, Issue 2, pp. 51-56. 2013. ISSN: 2210-8335. URL: http://www.sciencedirect.com/science/article/ pii/S2210833513000038. Fecha de consulta: 12-03-2018. DOI: 10.1016/j.jcgg.2012.11.004

[13] M.A. García Veiga. "Análisis causal con ecuaciones estructurales de la satisfacción ciudadana con los servicios municipales". Tesis para optar por el título de Máster en Técnicas Estadísticas. Facultad de Matemáticas. Universidad de Santiago de Compostela, Santiago de Compostela, España. 2011.

[14] V. Calás Pita. "Estudio de la calidad de vida del reparto Pedro Pompa centro en la ciudad de Bayamo". Granma Ciencia. Vol. 12 No 1, pp. 1-5. Bayamo, Cuba. 2008. ISSN: 1027-975X.

[15] S. Boffill Vega. "Modelo general para contribuir al desarrollo local, basado en el conocimiento y la innovación. Caso Yaguajay". Tesis para optar por el título de Doctor en Ciencias Técnicas. Facultad de Ingeniería Industrial. Universidad de Matanzas "Camilo Cienfuegos". Matanzas, Cuba. 2010.

[16] A.E. González Rodríguez. "Percepción de la Calidad de Vida Urbana en las ciudades de la Frontera Norte de México". Tesis para optar por el título de Máster en Ciencias. Colegio de la Frontera, Tijuana, México. 2008.
[17] C.A. Torres Tovar. "Calidad de vida: realidad y percepción”. Bitácora Urbano Territorial. Vol. $17 \mathrm{~N}^{\circ}$ 2, pp. 7-12. 2010. ISSN: 18890-61532-1. URL: https://revistas. unal.edu.co/index.php/bitacora/article/ download/18890/19781. Fecha de consulta: 10-05-2018. DOI: 10.15446/bitacora.

[18] I. Ebrahimzadeh, A.A. Shahraki, A.A. Shahnaz and A.M. Myandoab. "Progressing Urban Development and Life Quality Simultaneously". City, Culture and Society. Vol. 7, Issue 3, pp. 186-193. 2016. ISSN: 1877-9166. DOI: 10.1016/j.ccs.2016.03.001

[19] S.M. Yusoff, F. Yusof and A.F. Arshad. "Effectiveness Form and Content of the Local Plan as a Tool for the Quality of Life in Urban Area". Procedia - Social and Behavioral Sciences. Vol. 222, Issue 23, pp. $897-$ 906. 2016. ISSN: 1877-0428. URL: http:// www.sciencedirect.com/science/article/pii/ S1877042816303056\#. Fecha de consulta: 1307-2017. DOI: 10.1016/j.sbspro.2016.05.226

[20] C. Discoli, I. Martini, G. San Juan, D. Barbero, L. Dicroce, C. Ferreyro and J. Esparza. "Methodology Aimed at Evaluating Urban Life Quality Levels.”. Sustainable Cities and Society. Vol. 10, Issue February 2014, pp. 140-148. 2014. ISSN: URL: http://sedici. unlp.edu.ar/bitstream/handle/10915/36411/ Methodology_aimed_at_evaluating_urban_ life_quality_levelsCarlos..pdf?sequence $=5$. Fecha de consulta: 12-03-2018.

[21] R.W. Marans. "Quality of Urban Life Studies: an Overview and Implications for Environment-Behaviour Research". Procedia - Social and Behavioral Sciences. Vol. 35, pp. 9-22. 2012. ISSN: 1877-0428. DOI: $10.1016 /$ j.sbspro.2012.02.058

[22] M. Muñoz Conde, O. Fresneda Bautista, L.I. Gallo Peña, J. Martínez Collantes, A. Moreno Rivas, H. Torres Arias y O. Villamizar Díaz. "Calidad de vida urbana y capacidad de pago en los hogares bogotanos". Alcaldía Mayor de Bogotá y Centro de Investigaciones para el Desarrollo. Facultad de Ciencias Económicas. Universidad Nacional de Colombia. Bogotá, Colombia. 2012.

[23] M. Brambilla, A. Michelangeli and E. Peluso. "Equity in the City: on Measuring Urban (Ine)quality of Life". Urban Studies. Vol. 50, Issue 16, pp. 3205-3224. 2013. ISSN: 1360- 
063X. URL: http://citeseerx.ist.psu.edu/ viewdoc/download?doi=10.1.1.841.1490\&r ep=rep1\&type=pdf. Fecha de consulta: 1203-2018. DOI: $10.1177 / 0042098013484539$

[24] P. Kladivo and M. Halás. "Quality of life in an Urban Environment: a Typology of Urban Units of Olomouc". Quaestiones Geographicae. Vol. 31, Issue 2, pp. 49-60. 2012. ISSN: 0137-477X. URL: https:// www.degruyter.com/downloadpdf/j/ quageo.2012.31.issue-2/v10117-0120018-4/v10117-012-0018-4.pdf. Fecha de consulta: 24-07-2018. DOI: 10.2478/ v10117-012-0018-4

[25] N. Grundmann. "Estudio de calidad de vida aplicando las sistemas de información geográfica en Araduey (Palencia)". Tesis para optar por el título de Máster en Tecnologías de Información Geográfica. Facultad de Geografía e Historia. Universidad Complutense de Madrid, Castilla y León, España. 2011.

[26] M.A. Mohit. "Objective Analysis of Variation in the Regional Quality of Life in Malaysia and its Policy Implications". Procedia - Social and Behavioral Sciences. Vol. 101, Issue 8, pp. 454-464. 2013. ISSN: 1877-0428. DOI: 10.1016/j.sbspro.2013.07.219.

[27] M. Shaterian, A. Oshnooi and M. Ganjipour. "Measuring the Degree of Life Quality Satisfaction of Old Neighborhoods of Aran and Bidgol County.". Urban - Regional Studies and Research Journal. Vol. 4, Issue 13, pp. 33-36. 2012. ISSN: 2252-0848. URL: http://citeseerx. ist.psu.edu/viewdoc/download?doi=10.1.1.898. 7583\&rep=rep1\&type=pdf. Fecha de consulta: 12-03-2018. DOI: 10.1.1.898.7583

[28] S.D. Arifwidodo. "Exploring the Effect of Compact Development Policy to Urban Quality of Life in Bandung, Indonesia". City, Culture and Society. Vol. 3, Issue 4, pp. 303-311. December, 2012. ISSN: 1877-9166. URL: http://cyber.sci-hub.tw/ MTAuMTAxNi9qLmNjcy4yMDEyLjE $x \operatorname{Lj} A w N w==/$ a rif widodo 2012 . pdf\#view=FitH. Fecha de consulta: 23-072018. DOI: 10.1016/j.ccs.2012.11.007

[29] E.O. Ibem and D. Amole. "Subjective Life Satisfaction in Public Housing in Urban Areas of Ogun State, Nigeria.”. Cities. Vol. 35, pp. 51-61. 2013. ISSN: 0264-2751. DOI: 10.1016/j.cities.2013.06.004
[30] A.M. Mostafa. "Quality of Life Indicators in Value Urban Areas: Kasr Elnile Street in Cairo.". Procedia - Social and Behavioral Sciences. Vol. 50, pp. 254-270. 2012. ISSN: 1877-0428. DOI: 10.1016/j.sbspro. 2012.08.032

[31] D. Węziak Białowolska. "Quality of Life in Cities - Empirical Evidence in Comparative European Perspective". Cities. Vol. 58, pp. 87-96. 2016. ISSN: 0264-2751. URL: http://twin.sci-hub.tw/3210fd9d5af0c05f5 b9ea17aa59943d8/wziakbiaowolska2016. pdf\#view=FitH. Fecha de consulta: 15-062018. DOI: 10.1016/j.cities.2016.05.016.

[32] M. Carbó Carreté, J. Guàrdia Olmos, C. Giné and R.L. Schalock. "A Structural Equation Model of the Relationship between Physical Activity and Quality of Life.'. International Journal of Clinical and Health Psychology. Vol. 16, Issue 2, pp. 147-156. 2016. ISSN: 1697-2600. DOI: 10.1016/j.ijchp.2015.11.001

[33] N. Cancino, C. González, I. Gallardo y C. Estrada. "Evaluación de un modelo de calidad de vida construido desde los datos". Acta Colombiana de Psicología. Vol. 19 $\mathrm{N}^{\circ}$ 1, pp. 297-309. 2016. ISSN: 01239155. URL: http://editorial.ucatolica.edu. co/ojsucatolica/revistas_ucatolica/index. php/acta-colombiana-psicologia/article/ download/987/1055. Fecha de consulta: 1506-2018. DOI: 10.14718/ACP.2016.19.1.13

[34] J.F. Hair, G. Thomas, M. Hult, C.M. Ringle and M. Sarstedt. "A Primer on Partial Least Squares Structural Equation Modeling (PLSSEM)". SAGE Publications, Inc. Vol. 1, pp. 1-243. United States of America. ISBN: 978-1-4522-1744-4. 2014.

[35] R. Hernández Sampieri, C. Fernández Collado y P. Baptista Lucio. "Metodología de la Investigación". McGraw-Hill. 2da Edición. Vol. 1, pp. 233-426. México. ISBN: 970-10-1899-0. 1998.

[36] J.F. Hair, R.E. Anderson, R.L. Tatham y W.C. Black. "Análisis Multivariante". PrenticeHall. 5ta Edición. Vol. 1 pp. 1-832. Madrid, España. ISBN: 13: 9788483220351. 1999.

[37] P.J. Curran, S.G. West and J.F. Finch. "The Robustness of Test Statistics to Nonnormality and Specification Error in Confirmatory Factor Analysis". Psychological Methods. Vol. 1, Issue 1, pp. 16-29. 1996. ISSN: 19391463. DOI: 1082-989X. 